\title{
Mechanisms for optical binding
}

\author{
David L. Andrews* and Luciana C. Dávila Romero \\ School of Chemical Sciences, University of East Anglia, \\ Norwich NR4 7TJ, United Kingdom
}

\begin{abstract}
The phenomenon of optical binding is now experimentally very well established. With a recognition of the facility to collect and organize particles held in an optical trap, the related term 'optical matter' has also been gaining currency, highlighting possibilities for a significant interplay between optically induced inter-particle forces and other interactions such as chemical bonding and dispersion forces. Optical binding itself has a variety of interpretations. With some of these explanations being more prominent than others, and their applicability to some extent depending on the nature of the particles involved, a listing of these has to include the following: collective scattering, laser-dressed Casimir forces, virtual photon coupling, optically induced dipole resonance, and plasmon resonance coupling. It is the purpose of this paper to review and to establish the extent of fundamental linkages between these theoretical descriptions, recognizing the value that each has in relating the phenomenon of optical binding to the broader context of other, closely related physical measurements.
\end{abstract}

Keywords: Optical binding, Casimir force, light scattering, optical traps, nanoparticles, self-assembly, quantum electrodynamics, nanofabrication

\section{INTRODUCTION}

Before the arrival of the laser, few could have anticipated the immense impact that optical forces would prove to exhibit in the physical and biological sciences. At scales ranging from a single cell right down to that of a single atom, the mechanical effects of optical fields can have profound effects and far-reaching significance. With the growth to maturity of a range of laser-based optical trapping techniques, one of the major developments in the last decade has been the facility to engineer a plurality of trap sites, to capture particles in pre-defined array geometries. Related to this has been the emergence of a new interest in optical binding, where optically modified particle-particle interactions dominate. Optical binding is an optomechanical effect exhibited by systems of micro- and nanoparticles, irradiated with suitably off-resonance laser light.[1,2] Physically distinct from standing-wave and other forms of holographic optical trap, the phenomenon arises as a result of an inter-particle coupling engaging with individual radiation modes, leading to optically induced modifications to their electrodynamic interactions.

Within a semiclassical approach, one can correctly surmise the physical existence of an optically induced force that exhibits a strongly damped oscillatory dependence on the separation of the particle pair. The sinusoidal electric field of monochromatic radiation produces motions in the charge distributions of the particles it encounters. Such motions lead to corresponding oscillatory electric dipoles, whose phase is determined by that of the radiation at each particle. For two particles in sufficiently close proximity, the interactions between their oscillating dipoles is evidently subject to the relative phase of the optical field at the two locations; it is also subject also to the sharp decline of such an interaction with distance, see Figure 1.

The result is not quite as simple as one might expect, however, even in the case of two spherical particles, because the electric field produced by an oscillating dipole has a relatively complex dependence on displacement $\mathbf{R}$ and orientation of the source dipole $\mu$, as delivered by the following equation; [3] ( p.411)

\footnotetext{
*david.andrews@physics.org

Optical Trapping and Optical Micromanipulation VI, edited by Kishan Dholakia, Gabriel C. Spalding, Proc. of SPIE Vol. 7400, 74001H · () 2009 SPIE · CCC code: 0277-786X/09/\$18 · doi: 10.1117/12.827983
} 


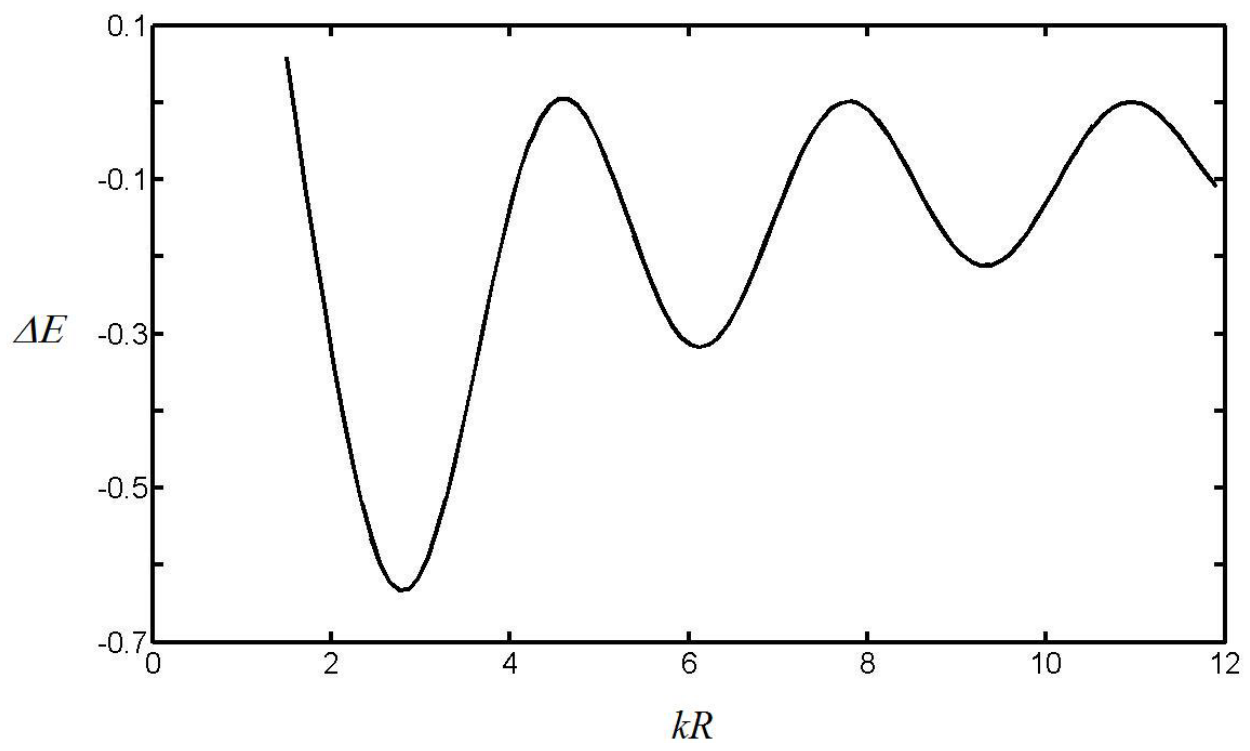

Fig 1. Dependence of optically-induced potential energy, for a pair of particles separated by distance $R$, plotted against $k R$, where $k=2 \pi / \lambda$ and $\lambda$ is the laser wavelength. The inter-particle axis is aligned with the propagation wave-vector direction, $\mathbf{k} \| \mathbf{R}$, and the polarization of the electric field is perpendicular to both.

$$
\mathbf{E}=k^{2}(\hat{\mathbf{R}} \times \boldsymbol{\mu}) \times \hat{\mathbf{R}} \frac{e^{\mathrm{i} k R}}{4 \pi \varepsilon_{0} R}+[3 \hat{\mathbf{R}}(\hat{\mathbf{R}} \cdot \boldsymbol{\mu})-\boldsymbol{\mu}]\left(\frac{1}{4 \pi \varepsilon_{0} R^{3}}-\frac{\mathrm{i} k}{4 \pi \varepsilon_{0} R^{2}}\right) e^{i k R}
$$

in which $k=\omega / c$ is the magnitude of the wave-vector $(2 \pi / \lambda)$ for an optical wavelength $\lambda$. In a medium of refractive index $n(\omega)$, equation (1) is modified by the replacement of each $k$ by $n(\omega) k$. While the above expression is fully consistent in form with the retarded field [4] that can be inferred from an analysis based on quantum electrodynamics, the latter theory affords deeper insights into the mechanisms at work in optical binding - and their relationship to dispersion forces.

The optically induced inter-particle interactions that result from each particle experiencing the retarded fields due to other neighboring particles give rise to forces and torques (usually described as optical binding, although the forces are not necessarily attractive in form). These forces and torques have been a particular focus in extensive recent investigations, see for example refs. [5-13] and citations therein. Such phenomena have increasingly been advocated as a tool for the optical manipulation and configuration of particles, and optically induced arrays have been observed in numerous experimental studies.[13-15] In this connection, the related term optical matter has also been coined, highlighting possibilities for a significant degree of interplay between optically induced inter-particle forces and other interactions such as chemical bonding and dispersion forces. Light-mediated inter-particle interactions undoubtedly offer the potential to organize large numbers of microparticles using optical force alone. Combined with established optical trapping techniques, this will undoubtedly prove one of the most important future directions in the field, particularly from the viewpoint of the physical sciences

In the research literature, the phenomenon of optical binding can be found attributed to a variety of mechanisms with apparently different interpretations. Some of these explanations have a greater currency than others, their applicability to a certain extent depending on the application, and often the constitution and form of the particles involved. Recognizing the value that each description has, especially in relating the phenomenon of optical binding to the broader context of trapping and closely related physical measurements, it is the purpose of this paper to review and to establish the extent of fundamental linkages between various theoretical descriptions. They prove to have much more in common than might be supposed. 


\section{QED BACKGROUND}

Quantum electrodynamics (QED) is a branch of theory that is rigorous and well-proven, offering a flexible and consistent methodology that is uniquely appropriate for addressing the quantum interactions of light and matter. It is, moreover, the framework that was used in formulating the first prediction of what has since become known as optical binding [16]. To establish the QED representation - which is characterized by dealing with both particles and the radiation field in a fully quantized setting - it is helpful to first recall how this theory addresses the normal dispersion interaction between particles. Here, calculations are performed on a system state in which both the particles and the radiation field are in the ground state, the latter exhibiting well-known vacuum fluctuations [17]. This system state couples with other short-lived states in which the electromagnetic field has a non-zero occupation number for one or more radiation modes, signifying the presence of virtual photons. The dispersion interaction emerges from a perturbative calculation based on an exchange of two virtual photons, each created at one particle and annihilated at the other. These two quanta of light may (but need not) overlap in time as they propagate between the two units. Cast in such terms, the theory delivers a result - the Casimir-Polder formula - that is valid for all distances. In correctly accounting for the retardation which leads to a long-range $R^{-7}$ asymptotic dependence on the pair separation $R$, [17] (p. 54) [18-23], this interpretation lends a fresh perspective to the physics involved in the more familiar $R^{-6}$ van der Waals interaction - which is the attractive part of the Lennard-Jones potential [24] (p.29). Such a development also strongly suggests that other effects may be manifest when the system state has an occupied radiation mode, i.e. when there is a throughput of electromagnetic radiation. Indeed it is the same, fourth order of perturbation theory that gives the leading result, and the optically conferred pair energies prove to exhibit a linear dependence on the photon number of the occupied mode. Cast in terms of experimental quantities, this is manifest as an energy shift $\Delta E_{\text {ind }}$, linearly proportional to the irradiance. The corresponding laser-induced coupling forces emerge from the potential energy result, as the spatial derivative.

We now consider in a little more detail the outcome of adopting, in the case of optical binding, this theoretical approach. $[2,9,25]$ The interactions of the electromagnetic field with two functionally identical particles $(A, B)$ are fundamentally based on electric interactions with the radiation field, expressible as follows (using the implied summation convention for repeated tensor and vector indices):

$$
H_{\mathrm{int}}^{\xi}=-\varepsilon_{o}^{-1} \sum_{\xi=A, B} \mu_{i}(\xi) d_{i}^{\perp}\left(\mathbf{R}_{\xi}\right)-\varepsilon_{o}^{-1} \sum_{\xi=A, B} Q_{i j}(\xi) \cdot \nabla_{j} d_{i}^{\perp}\left(\mathbf{R}_{\xi}\right)-\ldots
$$

$\mathbf{R}_{\xi}$ being the position vector of a dielectric particle $\xi$, with $\mu(\xi)$ and $\mathbf{Q}(\xi)$ the corresponding operators for the electric dipole and electric quadrupole (E1 and E2, respectively). The potential involvement of coupling with the magnetic field of the radiation is to be briefly discussed later in this Section. The second term in (1), which for radiation in the $\mathrm{uv} / \mathrm{visible}$ range is typically smaller than the first by the order of the fine structure constant (i.e. two-three orders of magnitude smaller), takes the lead in a series of higher order multipole corrections; for the present, only this leading correction is retained, with a view to the subsequent analysis. The electric displacement vector field $\mathbf{d}^{\perp}$ in (1) is itself expressible, using the Power-Zienau-Woolley approach, as a mode expansion that is linear in the photon creation and annihilation operators [26]. QED delivers a result applicable to optical fields with arbitrary polarization and beam geometry [2, 27, 28]. A suitable basis set for calculating the optical coupling, induced by throughput radiation, comprises products of the electronic states of each particle and a number state for each mode of the radiation field. The 'initial' and 'final' states of the optical binding system can be written as;

$$
\begin{aligned}
& \mid \text { initial }\rangle=\left|E_{0}^{A}, E_{0}^{B} ; n(\mathbf{k}, \lambda)\right\rangle, \\
& \mid \text { final }\rangle=\left|E_{0}^{A}, E_{0}^{B} ; n(\mathbf{k}, \lambda)\right\rangle .
\end{aligned}
$$

Here the equivalence of the two system state vectors signifies that diagonal elements of the transition matrix are to be derived - including non-trivial correction terms that arise from a coupling of off-diagonal matrix elements. The result is therefore to be interpreted as an energy (shift). As can be seen from expression (3), each particle $A$ and $B$ is generally considered to be in its lowest energy state, i.e. the ground state, remaining in this state beyond any interaction with the 
throughput radiation. Four possible mechanisms invite consideration: $(a)$ a case where photon absorption occurs in centre $A$, and photon emission into the same throughput mode occurs at centre $B ;(b)$ a mirror case where the roles of $A$ and $B$ are exchanged; $(c)$ and $(d)$ are cases involving a static form of contribution, in which both the photon absorption and emission events occur at the same location, either $A$ or $B$, see Figure 2. In the detailed quantum electrodynamical calculations, each of these mechanisms is associated with a set of quantum amplitudes differing in the time sequences of the interactions that are involved.

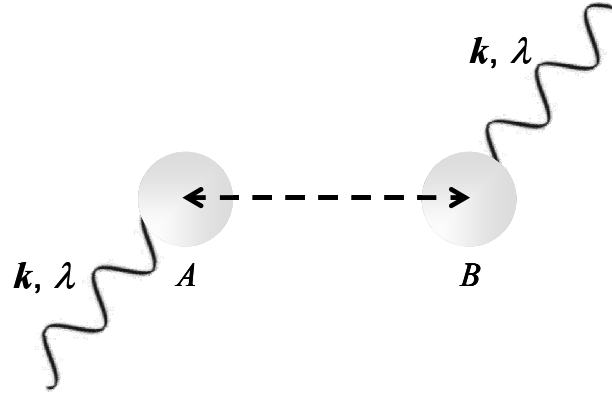

(a)

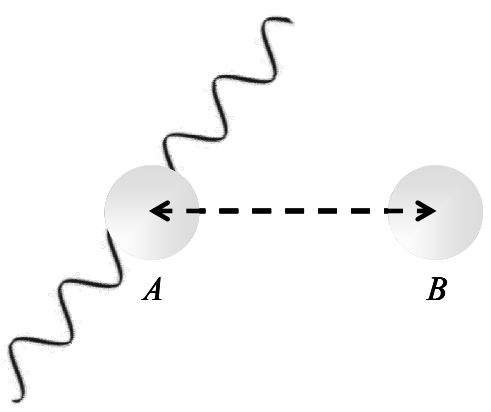

$(c)$

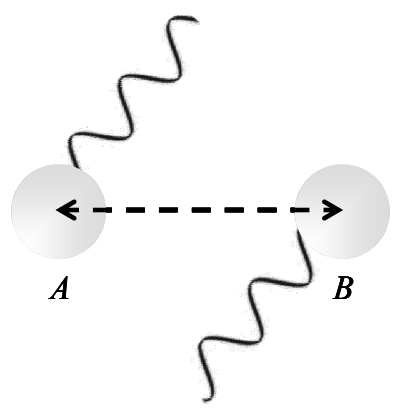

(b)

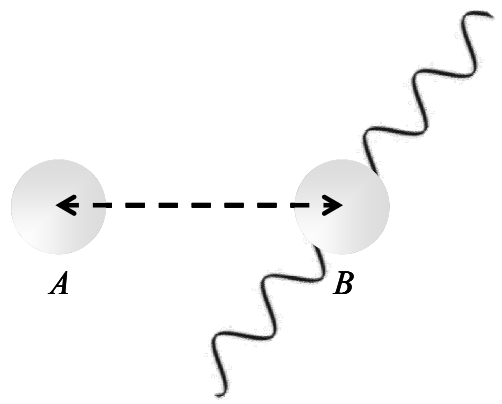

(d)

Fig 2. Four contributory mechanisms for optical binding. In the dynamic cases $(a)$ and $(b)$, the coupling depends on the polarizability of both particles. In $(a)$ the photon absorption occurs in centre $A$ and photon emission at $B$, while the process in $(b)$ is its mirror-image. In the static coupling of $(c)$ and $(d)$ the permanent dipole of one center is coupled to the hyperpolarizability of the other one.

After performing lengthy calculations, and casting the result in terms of physically measurable quantities, the following result emerges for the dominant term in the optically induced shift in inter-particle potential energy, produced by a beam of irradiance $I[1]$;

$$
\begin{aligned}
& \Delta E_{i n d}(\mathbf{k}, \mathbf{R})=\left(\frac{I}{\varepsilon_{0} c}\right) e_{i}^{(\lambda)} \bar{e}_{l}^{(\lambda)} \operatorname{Re}\left\{\alpha_{i j}^{A}(k) V_{j k}(k, \mathbf{R}) \alpha_{k l}^{B}(k) \exp (-i \mathbf{k} \cdot \mathbf{R})\right. \\
& \left.\quad+\alpha_{i j}^{B}(k) V_{j k}(k, \mathbf{R}) \alpha_{k l}^{A}(k) \exp (i \mathbf{k} \cdot \mathbf{R})+\frac{1}{2} V_{j k}(0, \mathbf{R})\left[\beta_{i j l}^{A}(k) \mu_{k}^{B}+\mu_{k}^{A} \beta_{i j l}^{B}(k)\right]\right\},
\end{aligned}
$$

where $\mathbf{R}$ is the separation between the particles, $\mathbf{R}_{B}-\mathbf{R}_{A}, e_{i}^{(\lambda)}$ is the $i^{\text {th }}$ component of the laser polarization vector, $\alpha_{i j}^{\xi}(k) \equiv \alpha_{i j}^{\xi}(-k ; k)$ is the frequency-dependent polarizability and $\beta_{i j l}^{\xi}(k) \equiv \beta_{i j l}^{\xi}(-k ; 0, k)$ the corresponding electro-optic hyperpolarizability of particle $\xi$ [26], and $V_{j k}(k, \mathbf{R})$ is the retarded resonance dipole-dipole interaction tensor [29]; 


$$
V_{i j}(k, \mathbf{R})=\frac{\exp (i k R)}{4 \pi \varepsilon_{0} R^{3}}\left[(1-i k R)\left(\delta_{i j}-3 \hat{R}_{i} \hat{R}_{j}\right)-(k R)^{2}\left(\delta_{i j}-\hat{R}_{i} \hat{R}_{j}\right)\right]
$$

No other contributions arise at this order $\left(E 1^{4}\right)$. The calculation that leads to (5) involves a summation over radiative modes for a virtual photon traveling in either direction between $A$ and $B$. The last two terms in equation (4) involve static dipole moments, $\boldsymbol{\mu}$, for each particle; each term relates to a process in which both the annihilation and re-creation of the beam photons are co-located at one or the other particle; these terms are independent of $k$ and simply represent an additional, repulsive correction that drops off with $R^{-3}$. Clearly, if both particles are centrosymmetric and non-polar, as will commonly be the case, these terms vanish. Even when that is not the case, these terms can only serve to modify the exact positions of the optical binding minima.

Before proceeding further we briefly address higher multipolar contributions to the induced energy shift $\Delta E_{\text {ind }}(\mathbf{k}, \mathbf{R})$ [30]. The next highest order contributions emerge when the second term in equation (1), the electric quadrupole interaction Hamiltonian, is considered as the coupling basis for any one of the four photon events described previously. Introducing the appropriate modification to the first two terms in equation (2), the additional (E1 $\left.{ }^{3} \mathrm{E} 2\right)$ contributions to the energy which result are given by;

$$
\begin{aligned}
& \Delta E_{i n d}^{(Q)}(k, \mathbf{R})=\left(\frac{I}{\varepsilon_{o} c}\right) \operatorname{Re}\left[e _ { i } ^ { ( \lambda ) } \overline { e } _ { l } ^ { ( \lambda ) } \left(i V_{j k}(k, \mathbf{R}) k_{m}\left(A_{i m j}^{\mathrm{A}}(k) \alpha_{k l}^{\mathrm{B}}(k)-\alpha_{i j}^{\mathrm{A}}(k) A^{\prime \mathrm{B}}(k m l)\right)\right.\right. \\
& \left.\left.+\alpha_{i j}^{\mathrm{A}}(k) V_{j k m}(k, \mathbf{R}) A^{\prime}{ }_{k m l}^{\mathrm{B}}(k)+A_{i j m}^{\mathrm{A}}(k) V_{j m k}(k, \mathbf{R}) \alpha_{k l}^{\mathrm{B}}(k)\right) \exp (-i \mathbf{k} \cdot \mathbf{R})\right] .
\end{aligned}
$$

Here, the term involving the dipole-dipole interaction tensor $V_{j k}(k, \mathbf{R})$ arises from contributions where one of the real, laser photon interactions is accomplished by an electric-quadrupole interaction. In the remainder of the above expression (6), it is the virtual coupling photon that experiences one quadrupole interaction, reflected in the involvement of the fully retarded resonance quadrupole-dipole interaction tensor $V_{i j k}(k, \mathbf{R})$, given by [31];

$$
\begin{aligned}
& V_{i j k}(k, \mathbf{R})= \\
& \frac{i e^{i k R}}{4 \pi \varepsilon_{o}}\left[\left(\hat{R}_{i} \hat{R}_{j} \hat{R}_{k}-\delta_{i j} \hat{R}_{k}\right)\left(\frac{k^{3}}{R}+\frac{i k^{2}}{R^{2}}\right)\right. \\
& \left.+\left(\delta_{i j} \hat{R}_{k}+\delta_{i k} \hat{R}_{j}+\delta_{j k} \hat{R}_{i}-5 \hat{R}_{i} \hat{R}_{j} \hat{R}_{k}\right)\left(\frac{i k^{2}}{R^{2}}+\frac{3 k}{R^{3}}+\frac{i 3}{R^{4}}\right)\right] .
\end{aligned}
$$

The frequency-dependent dipole-quadrupole polarizabilities $A_{i m k}^{\xi}(k)$ and $A^{\prime} \xi_{i j k}^{\xi}(k)$ for particle $\xi$ are expressible as;

$$
\begin{aligned}
& A_{i m k}^{\xi}(k)=\sum_{s}\left[\frac{\mu_{i}^{0 s}(\xi) Q_{m k}^{s 0}(\xi)}{\left(E_{s 0}-\hbar c k\right)}+\frac{Q_{m k}^{0 s}(\xi) \mu_{i}^{s 0}(\xi)}{\left(E_{s 0}+\hbar c k\right)}\right], \\
& A^{\prime} \xi(k)=\sum_{s i j k}\left[\frac{Q_{i j}^{0 s}(\xi) \mu_{k}^{s 0}(\xi)}{\left(E_{s 0}-\hbar c k\right)}+\frac{\mu_{k}^{0 s}(\xi) Q_{i j}^{s 0}(\xi)}{\left(E_{s 0}+\hbar c k\right)}\right] .
\end{aligned}
$$

The general form of these results is an $\mathrm{E} m^{2} \mathrm{E} n^{2}$ shift, for arbitrary integers $m$ and $n$. In passing, we note that the expression obtained in equation (3.2) of reference [28] and given as the lowest order correction is a result calculated for a case involving two electric-quadrupole events. However, symmetry permitting, expression (4) is the lowest order correction to the energy because it invokes only one quadrupole interaction. The odd parity rank 3 tensors $\mathbf{A}^{\xi}$ and $\mathbf{A}^{\prime \xi}$ vanish if the particles are centrosymmetric. Crucially, any dipolar particle (i.e. one whose static electric dipole moment 
is non-zero) will also have non-zero $\boldsymbol{\beta}^{\xi}$ tensors. Hence, the third and fourth terms in equation (2) will also contribute and since they have $\mathrm{E} 1^{4}$ form, they will outweigh any contribution involving an electric quadrupole. For completeness, it is also important to note that the leading corrections produced by the involvement of a magnetic-dipole interaction are of the same order as the electric quadrupole correction [32] and therefore they, too, can be disregarded in the following study of the optically induced interaction energy shift.

In the following, consistent with the majority of experimental studies, we focus on spherical particles. Nonetheless it is notable that QED calculations have also been performed for non-spherical particles - where the parameter space is substantially larger. One potentially important application is where particles have cylindrical symmetry, their polarizability being characterized by one transverse and one longitudinal component. The corresponding results, for cylindrical particles in various configurations, have been applied to single-walled carbon nanotubes. These nanoparticles are of interest not only for their intrinsic properties and applications but, since they are strongly polarizable species, they afford ideal opportunities to exploit the quadratic dependence on polarizability in the potential energy equations. Assuming that the $\alpha_{\perp}$ and $\alpha_{\square}$ values are consistent with the corresponding static polarizabilities, then for nanotubes 200 $\mathrm{nm}$ in length and $0.4 \mathrm{~nm}$ in radius, separated by a distance $R=2 \mathrm{~nm}$, and with an incident intensity $I=1 \times 10^{16} \mathrm{~W} \mathrm{~m}^{-2}$, the forces range between $10^{-12}$ and $10^{-5} \mathrm{~N}$, according to the geometry [33]. The detailed results again reinforce the observation that laser-induced forces can be either positive or negative according to conditions; use of the term 'binding' force in various papers $[25,34]$ is potentially misleading, though ingrained. To quantify the forces it might prove possible to deploy atomic force microscopy - a technique already associated with carbon nanotubes [35]. Significantly, the forces lie in a range indicating realistic possibilities for the nanomanipulation of carbon nanotubes, through laser optomechanical control. Other studies of optical trapping and binding of cylindrical particles have been carried out by Grzegorczyk et al. [36, 37].

\section{OPTICAL BINDING MECHANISMS}

A singular advantage of the QED representation developed above is that is provides a rigorous basis on which to compare the variety of other mechanistic interpretations of optical binding. Amongst the most commonly invoked mechanisms one has to include: laser-dressed Casimir forces, optically induced dipole resonance, collective scattering, virtual photon coupling, and plasmon resonance coupling.

Laser-dressed Casimir forces: It has been shown that the origins of optical binding lie in a form of radiation-induced coupling representing an extension to the usual Casimir-Polder interactions - the latter signifying dispersion interactions in a form that accommodates the effects of retardation. In the absence of populated radiative modes, Casimir-Polder interactions entail the pairwise exchange of virtual photons [38], i.e. two virtual photon creation events and two corresponding annihilations. (The reader is referred elsewhere [20] for a concise alternative QED representation, also based on QED, but closer in spirit to the classical representation). When laser light is present, one or more radiation modes has a non-zero occupation number, and any photon from these modes may take the place of one virtual photon generating intensity-dependent additional terms. Conversely, the Casimir-Polder interaction - even the London interaction which is its short-range asymptote - can thus be understood as a special case of optical binding where the radiation field comprises only vacuum fluctuations. This much is evident from the form of the state vectors (2), which reduce to the basis for Casimir forces once the occupation number of 'throughput' radiation is equated to zero. The corollary is that the phenomenon of optical binding essentially subsumes the normal dispersion interaction - one important physical consequence being that it can over-ride the latter at routinely attainable levels of light intensity.

Optically induced dipole resonance: The classical description of light scattering depicts the oscillatory electric field of incoming radiation inducing a similarly fluctuating electric dipole moment, a process that occurs irrespective of the presence or absence of any intrinsic, static dipole. The dynamic polarizability thereby represents a constant of proportionality between the induced dipole and the strength of the electric field. Moreover, the fluctuating electric dipole is interpreted as the source of the scattered light, in the usual sense of a radiating dipole. Crucially, this model applies not only to deflective light scattering - it also applies to coherent, forward Rayleigh scattering where the emergent light proceeds onwards in phase with the throughput radiation. When two particles in sufficiently close proximity undergo the same process, it can be anticipated that a coupling will occur between the induced electric dipole moments that each experiences. At short distances this coupling will take precisely the same form as that which applies 
to the interaction between static dipoles; at longer distances a retardation effect will modify the form of the coupling. Using the textbook formulation of retarded dipolar fields [3] pp411, it is readily shown that precisely the same result as is given by equations (3) and (4) emerges, see Figure 2(a) and (b) .

Collective scattering: Most of the theoretical studies on optical binding concentrate on the optical interaction between two particles. However, experimentally the phenomena has been observed in multi-particles systems, where it has been shown that optical binding facilitates the formation of arrays in particular geometries [39, 40], and there is scope for controlled optical manipulation [41]. It is apparent that the principles of interaction between a pair of spherical particles afford a tractable basis for extension to a system of $N$ such particles. In general, a determination of the optically induced potential experienced by the whole assembly requires the evaluation of equation (4) for $N ! /(N-2) ! / 2$ particle pairs obviously making an analysis of the system significantly more complex when considering a large number of particles. Nevertheless, considering the possibility of optically fabricating structures for the next simplest case of just three particles is an interesting and expedient way to approach the problem. Such a system represents a key prototype for developing generic models of multi-particle complexity [30]. Additional, higher-order contributions to the opticallyinduced energy shift can be calculated from first principles. A system involving $N$ particles will accommodate $N$ ! contributory mechanisms, each one involving calculations based on $(2 N)$ ! time-ordered diagrams - for example, in the case of a three-particle system this would involve consideration of 6 different mechanisms, each involving 720 time orderings. Fortunately the coupling method introduced in [42] offers a straightforward answer to such a problem. This method can deliver a result for a system with an unspecified number of particles, $N$.

Virtual photon coupling: Virtual photons can be understood as 'borrowing' energy from the vacuum, consistent with the exploitation of an energy uncertainty $h / t$, where $t$ is the photon time-of-flight (here, determined by the distance between the two particles). This signifies a temporary relaxation of exact energy conservation in the isolated photon creation and annihilation events. When the system within which the virtual photon propagates enters its final state, i.e. after the virtual photon is extinguished, energy conservation is restored. Another key feature of virtual photon behavior is that only photons whose propagation vector $\mathbf{p}$ is essentially collinear to $\mathbf{R}$ remain significant as the inter-particle separation increases. In contrast, in the short-range region where $k R<<1$, photons with $\mathbf{p}$ not parallel to $\mathbf{R}$ are also involved in the coupling. This again is consistent with the position-momentum uncertainty principle, i.e. the positions of the photon creation and annihilation events are uncertain. See, for example, [4].

Plasmon resonance coupling: Laser-irradiated metal nano-structures give rise to locally enhanced fields, associated with high field gradients due to their strong spatial confinement [43]. Consequently several proposals have been made to apply these gradients in practical methods of nanomanipulation. Compared to dielectrics, metallic particles differ in several respects; put simply, per unit volume they present much larger polarizability values. To accurately model the optical forces exerted on metal nanoparticles it is however necessary to consider the associated dispersion properties. Specifically, in the Drude model, the frequency-dependence of the polarizability in (4) emerges from the formula $m^{2}=1-\left\{\omega_{p}^{2} /\left(\omega^{2}+i \gamma \omega\right)\right\}$, where $\omega_{p}$ is the plasmon frequency and $\gamma$ is the damping. It also becomes important to consider that the behavior of the conduction electrons alters, when they are in close proximity to the metal surface. If the particle dimensions are smaller than the corresponding 'skin depth', the incident field interacts with the whole particle volume, resulting in a linear dependence between force and volume - the particle is rather transparent. However, for particle sizes greater than the skin depth, the field interacts only with a thin outer layer and the force varies as the square of the diameter.

\section{DISCUSSION}

This paper has exhibited the variety of current interpretations for optical binding, listing a number of mechanisms which are ultimately proven to be different facets of the same phenomenon. A QED representation provides a rigorous basis on which such mechanisms can be linked and their value established in the context of closely related physical measurements. For completeness, one other factor deserves mention; it is worth observing that the exact positioning of minima in the potential energy landscapes is influenced by contributions that can be associated with optically induced dc fields. This is connected with a process known as optical rectification, [44-46] which is known to be responsible for the production of a dc electric field on the passage of laser light through suitably non-centrosymmetric media. In a similar 
spirit to the classical picture of induced dipole coupling, optically induced forces arise between two particles in close proximity, due to such induced dc fields. In essence, the static electric field that is generated when one particle mediates a process of optical rectification will interact with any static moment possessed by a neighboring particle. Here, no retardation operates; the interaction has the inverse cubic dependence on particle separation that characterizes the standard dipole-dipole coupling formula. In QED language, this relates directly to the mechanisms shown in Figure 2(c) and $(d)$. Although such interactions cannot engender local minima in the potential energy landscapes, they do serve to modify the positions of minima arising from the other mechanisms discussed above.

\section{ACKNOWLEDGEMENTS}

The authors gratefully acknowledge support from the Engineering and Physical Sciences Research Council, also thanking Justo Rodríguez for kindly supplying one of the figures for this paper.

\section{REFERENCES}

[1] D. S. Bradshaw, and D. L. Andrews, "Optically induced forces and torques: Interactions between nanoparticles in a laser beam," Physical Review A, 72(3), 033816 (2005).

[2] L. C. Dávila Romero, and D. L. Andrews, "Nanoscale Optics: Interparticle Forces" in [Structured Light and Its Applications: An Introduction to Phase-Structured Beams and Nanoscale Optical Forces] Academic Press, Burlington MA(2008).

[3] J. D. Jackson, [Classical Electrodynamics] Wiley, New York ; Chichester, (1999).

[4] D. L. Andrews, and D. S. Bradshaw, "Virtual photons, dipole fields and energy transfer: a quantum electrodynamical approach,” European Journal of Physics, 25(6), 845-858 (2004).

[5] M. Guillon, O. Moine, and B. Stout, "Longitudinal optical binding of high optical contrast microdroplets in air," Physical Review Letters, 96(14), 143902 (2006).

[6] A. Salam, "On the effect of a radiation field in modifying the intermolecular interaction between two chiral molecules," Journal of Chemical Physics, 124(1), 014302 (2006).

[7] A. Salam, "Intermolecular interactions in a radiation field via the method of induced moments," Physical Review A (Atomic, Molecular, and Optical Physics), 73(1), 013406 (2006).

[8] A. Salam, "Two alternative derivations of the static contribution to the radiation-induced intermolecular energy shift," Physical Review A (Atomic, Molecular, and Optical Physics), 76(6), 063402 (2007).

[9] D. L. Andrews, R. G. Crisp, and D. S. Bradshaw, "Optically induced inter-particle forces: from the bonding of dimers to optical electrostriction in molecular solids," Journal of Physics B-Atomic Molecular and Optical Physics, 39(15), S637-S650 (2006).

[10] T. M. Grzegorczyk, B. A. Kemp, and J. A. Kong, "Stable optical trapping based on optical binding forces," Physical Review Letters, 96(11), 113903 (2006).

[11] L. C. Dávila Romero, J. Rodríguez, and D. L. Andrews, "Electrodynamic mechanism and array stability in optical binding," Optics Communications, 281, 865-870 (2008).

[12] J. Rodríguez, L. C. Dávila Romero, and D. L. Andrews, "Optical binding: Potential energy landscapes and QED," Proc. SPIE 6905, 69050H.

[13] R. F. Marchington, M. Mazilu, S. Kuriakose et al., "Optical deflection and sorting of microparticles in a nearfield optical geometry,” Optics Express, 16(6), 3712-3726 (2008).

[14] S. K. Mohanty, J. T. Andrews, and P. K. Gupta, "Optical binding between dielectric particles," Optics Express, 12(12), 2746-2753 (2004). 
[15] C. D. Mellor, T. A. Fennerty, and C. D. Bain, "Polarization effects in optically bound particle arrays," Optics Express, 14(21), 10079-10088 (2006).

[16] T. Thirunamachandran, "Intermolecular interaction in the presence of an intense radiation-field," Molecular Physics, 40(2), 393-399 (1980).

[17] P. W. Milonni, [The Quantum Vacuum: An Introduction to Quantum Electrodynamics] Academic Press, Boston, Mass. ; London, (1994).

[18] H. B. G. Casimir, and D. Polder, "The Influence of Retardation on the London-Van der Waals Forces," Physical Review, 73(4), 360-372 (1948).

[19] D. L. Andrews, and L. C. Dávila Romero, "Conceptualization of the Casimir effect," European Journal of Physics, 22(4), 447-451 (2001).

[20] E. A. Power, "Casimir-Polder potential from first principles," European Journal of Physics, 22(4), 453-461 (2001).

[21] G. J. Maclay, H. Fearn, and P. W. Milonni, "Of some theoretical significance: implications of Casimir effects," European Journal of Physics, 22(4), 463-469 (2001).

[22] B. W. Alligood, and A. Salam, "On the application of state sequence diagrams to the calculation of the CasimirPolder potential,” Molecular Physics, 105(4), 395-404 (2007).

[23] F. Capasso, J. N. Munday, D. Iannuzzi et al., "Casimir forces and quantum electrodynamical torques: Physics and nanomechanics," IEEE Journal of Selected Topics in Quantum Electronics, 13(2), 400-414 (2007).

[24] A. Altland, and B. Simons, [Condensed Matter Field Theory] Cambridge University Press, Cambridge ; New York, (2006).

[25] F. Depasse, and J. M. Vigoureux, "Optical Binding Force between 2 Rayleigh Particles," Journal of Physics DApplied Physics, 27(5), 914-919 (1994).

[26] D. P. Craig, and T. Thirunamachandran, [Molecular Quantum Electrodynamics: An Introduction to RadiationMolecule Interactions] Dover Publications, Mineola, NY, (1998).

[27] D. S. Bradshaw, and D. L. Andrews, "Optically induced forces and torques: Interactions between nanoparticles in a laser beam (vol 72, 033816, 2005)," Physical Review A, 73(3), 039903 (2006).

[28] A. Salam, "Intermolecular interactions in a radiation field via the method of induced moments," Physical Review A, 73(1), 013406 (2006).

[29] G. J. Daniels, R. D. Jenkins, D. S. Bradshaw et al., "Resonance energy transfer: The unified theory revisited," Journal of Chemical Physics, 119(4), 2264-2274 (2003).

[30] J. Rodríguez, L. C. Dávila Romero, and D. L. Andrews, "Optical binding in nanoparticle assembly: Potential energy landscapes," Physical Review A (Atomic, Molecular, and Optical Physics), 78(4), 043805-7 (2008).

[31] G. D. Scholes, and D. L. Andrews, "Damping and higher multipole effects in the quantum electrodynamical model for electronic energy transfer in the condensed phase," Journal of Chemical Physics, 107(14), 5374-5384 (1997).

[32] D. L. Andrews, and P. Allcock, [Optical Harmonics in Molecular Systems] Wiley-VCH, Weinheim; Great Britain, (2002).

[33] D. L. Andrews, and D. S. Bradshaw, "Laser-induced forces between carbon nanotubes," Optics Letters, 30(7), 783-785 (2005).

[34] P. C. Chaumet, and M. Nieto-Vesperinas, "Optical binding of particles with or without the presence of a flat dielectric surface," Physical Review B, 64(3), 035422 (2001).

[35] L. Guo, J. Liang, S. Dong et al., "Property of carbon nanotube tip for surface topography characterization," Applied Surface Science, 228(1-4), 53-56 (2004). 
[36] T. M. Grzegorczyk, B. A. Kemp, and J. A. Kong, "Stable optical trapping based on optical binding forces," Physical Review Letters, 96(11), 113903 (2006).

[37] T. M. Grzegorczyk, B. A. Kemp, and J. A. Kong, "Trapping and binding of an arbitrary number of cylindrical particles in an in-plane electromagnetic field," Journal of the Optical Society of America A-Optics Image Science and Vision, 23(9), 2324-2330 (2006).

[38] E. A. Power, [Introductory Quantum Electrodynamics] Longmans, London, 13 and 131 (1964).

[39] N. K. Metzger, E. M. Wright, W. Sibbett et al., "Visualization of optical binding of microparticles using a femtosecond fiber optical trap," Optics Express, 14(8), 3677-3687 (2006).

[40] M. Guillon, and B. Stout, "Optical trapping and binding in air: Imaging and spectroscopic analysis," Physical Review A (Atomic, Molecular, and Optical Physics), 77(2), 023806-8 (2008).

[41] R. F. Marchington, M. Mazilu, S. Kuriakose et al., "Optical deflection and sorting of microparticles in a nearfield optical geometry,” Optics Express, 16(6), 3712-3726 (2008).

[42] L. C. Dávila Romero, and D. L. Andrews, "A retarded coupling approach to intermolecular interactions," Journal of Physics B-Atomic Molecular and Optical Physics, 42(8), 10 (2009).

[43] L. Novotny, and C. Henkel, "Van der Waals versus optical interaction between metal nanoparticles," Optics Letters, 33(9), 1029-1031 (2008).

[44] M. Bass, P. A. Franken, J. F. Ward et al., “Optical Rectification,” Physical Review Letters, 9(11), 446 (1962).

[45] M. Bass, P. A. Franken, and J. F. Ward, "Optical Rectification," Physical Review, 138(2A), A534 (1965).

[46] L. C. Dávila Romero, S. Naguleswaran, G. E. Stedman et al., "Electro optic rotation in isotropic media," Nonlinear Optics, 23, 191-201 (2000). 